J. Beerten, D. Van Hertem, and R. Belmans, "VSC MTDC systems with a distributed DC voltage control - a power flow approach," Proc. IEEE PowerTech 2011, Trondheim, Norway, Jun. 19-23, 2011, 6 pages.

Digital Object Identifier: 10.1109/PTC.2011.6019434

URL:

http://ieeexplore.ieee.org/xpl/articleDetails.jsp?tp=\&arnumber=6019434

(C) 2011 IEEE. Personal use of this material is permitted. Permission from IEEE must be obtained for all other users, including reprinting/ republishing this material for advertising or promotional purposes, creating new collective works for resale or redistribution to servers or lists, or reuse of any copyrighted components of this work in other works. 


\title{
VSC MTDC Systems with a Distributed DC Voltage Control - A Power Flow Approach
}

\author{
Jef Beerten, Student Member, IEEE, Dirk Van Hertem, Senior Member, IEEE, and Ronnie Belmans, Fellow, IEEE
}

\begin{abstract}
In this paper, a power flow model is presented to include a DC voltage droop control or distributed DC slack bus in a Multi-terminal Voltage Source Converter High Voltage Direct Current (VSC MTDC) grid. The available VSC MTDC models are often based on the extension of existing point-to-point connections and use a single DC slack bus that adapts its active power injection to control the DC voltage. A distributed DC voltage control has significant advantages over its concentrated slack bus counterpart, since a numbers of converters can jointly control the DC system voltage. After a fault, a voltage droop controlled DC grid converges to a new working point, which impacts the power flows in both the DC grid and the underlying AC grids. Whereas current day research is focussing on the dynamic behaviour of such a system, this paper introduces a power flow model to study the steady-state change of the combined AC/DC system as a result of faults and transients in the DC grid. The model allows to incorporate DC grids in a $\mathrm{N}-1$ contingency analysis, thereby including the effects of a distributed voltage control on the power flows in both the AC and DC systems.
\end{abstract}

Index Terms-DC Grids, HVDC transmission, Load flow analysis, Voltage control.

\section{INTRODUCTION}

A $\mathrm{T}$ present, the power engineering world is facing enormous challenges. Today's power systems are operated more closely to their limits, while system operators are more and more faced with an increased public opposition to the construction of new lines. The projected massive integration of intermittent renewable energy sources also imposes major technical challenges in terms of a secure grid operation.

The growing need for transmission capacity that accompanies the recent challenges has lead to an increased interest in High Voltage Direct Current (HVDC) systems in multi-terminal (MTDC) configuration as an alternative to grid enforcements based on AC technology. In Europe, suggestions are even made to construct a whole new overlaying DC 'supergrid', as DC technology has technical and economical advantages over traditional AC transmission. The meshed DC supergrid could thereby interconnect remotely located offshore wind farms and connect them with various points in the existing AC infrastructure, to provide a more reliable grid. Special attention is given to HVDC based on Voltage Source Converter (VSC) technology as it has significant advantages over

Jef Beerten is funded by a research grant from the Research Foundation Flanders (FWO).

The authors are with the Department of Electrical Engineering (ESAT), Division ELECTA, Katholieke Universiteit Leuven, Kasteelpark Arenberg 10, bus 2445, 3001 Leuven-Heverlee, Belgium (e-mail: jef.beerten@esat.kuleuven.be, dirk.vanhertem@ieee.org, both Line-Commutated Converters (LCC) and traditional AC technology. Contrary to LCC, the VSC technology can support the AC grid due to a fast and independent control of active and reactive power. The inherent technical characteristics of VSCs give the technology much better prospects for a multiterminal operation than its LCC counterpart. Furthermore, a connection to remote wind farms would only be realistic with VSC technology. As European plans and studies to gradually construct such an overlaying DC grid are taken more concrete forms, the technical problems yet still unsolved are becoming predominantly important in current day research on DC grids.

An outstanding research issues is a distributed control of the system voltage in such a DC grid. A strong urge has risen in recent years to thoroughly address this issue and to provide robust control functions to increase the overall safety of the DC grid [1]. An extension of the control principles of pointto-point connections gives rise to one converter controlling the DC voltage at its terminals while the other converters control their active power injections in the AC grid. The DC voltage controlling converter is often referred to as the 'DC slack' converter since the control adapts the output power automatically to compensate for the losses in the DC system. Such a centralised DC slack converter has to react fast on DC grid transients, such as e.g. the loss of converters or DC line. The converter needs to be oversized and connected to a strong AC system to cope with severe system transients. Furthermore, an outage of this converter cannot be covered. The control function of the slack bus converter can be duplicated to other converters that would be functioning as a back-up slack to take over the DC voltage control in case the primary DC slack converter fails. While a duplication could increase the overall reliability of the system, it does not disregard the main disadvantages of a centralised DC slack converter [2]. Beside the technical problems, the geographic location of such a DC slack converter might be controversial, as one system operator would have to cope with all problems on the DC grid.

Alternatively to a centralised DC slack approach, the DC voltage control can be distributed over a number of converters using a voltage droop control [3], [4]. In this way, a number of converters contribute to the control of the DC system voltage by adopting their active power input when the DC system voltage alters as a result of changes in the operation of the grid. Whereas previous research has primarily focused on the transient behaviour of a VSC MTDC systems with a distributed voltage control [5], [6], the steady-state behaviour of distributed voltage control schemes and its integration in power flow algorithms has mainly remained unaddressed so far. However, when system studies and N-1 contingency analyses of systems including DC grids need to be undertaken, 


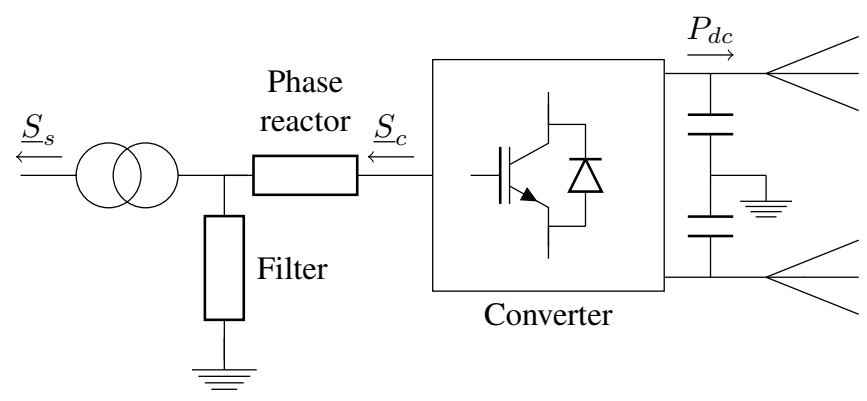

Fig. 1. VSC HVDC converter station connected to a DC grid.

the steady-state power flows and the effect of this distributed voltage control are of primary interest [7]. Contrary to the situation in case of a system with a centralised slack bus, a converter outage will give rise to a change of the power setpoints of all voltage controlling converters. This paper aims to provide an answer to this mainly unaddressed research question by presenting a model to fully integrate a distributed voltage control in an AC/DC power flow program.

The paper elaborates further on the sequential AC/DC power flow algorithm presented in [8] and discusses how the algorithm has to be extended to include the effects of a distributed DC voltage control on the power flows. Section II briefly discusses the control principles of VSC HVDC converters that are of interest with regard to power flow algorithms. Special attention is paid to the proposed voltage droop control strategy. Section III discusses the representation of the DC grid and Section IV presents the inclusion of the distributed voltage control in the AC/DC power flow algorithm. Section V shows the effects of a converter outage on the power flows by means of simulation results.

\section{VSC CONVERTER CONTROL}

As addressed in the previous section, a VSC HVDC converter, shown in Fig. 1, can fully control its apparent power injection by independently controlling its active and reactive power, respectively $P_{s}$ and $Q_{s}$. To achieve this, a vectorcontrol scheme with two inner current controllers independently controls the converter currents in a rotating $d q$-reference frame. Slower outer control loops allow to independently control the active and reactive power injections by changing the reference set-points of the inner current control loops.

From a power flow point-of-view, only the steady-state behaviour of the outer control loops is of importance. A VSC converter can exhibit two reactive power control functions:

1) $Q$-control: The reactive power $Q_{s}$ injected in the $\mathrm{AC}$ grid is kept constant.

2) $U$-control: The converter adopts its reactive power injection $Q_{s}$ to keep its AC bus voltage magnitude $U_{s}$ constant.

As far as active power is concerned, current-day twoterminal VSC HVDC transmission schemes have two different control functions for each converter:

1) P-control: The active power $P_{s}$ injected in the AC grid is kept constant.

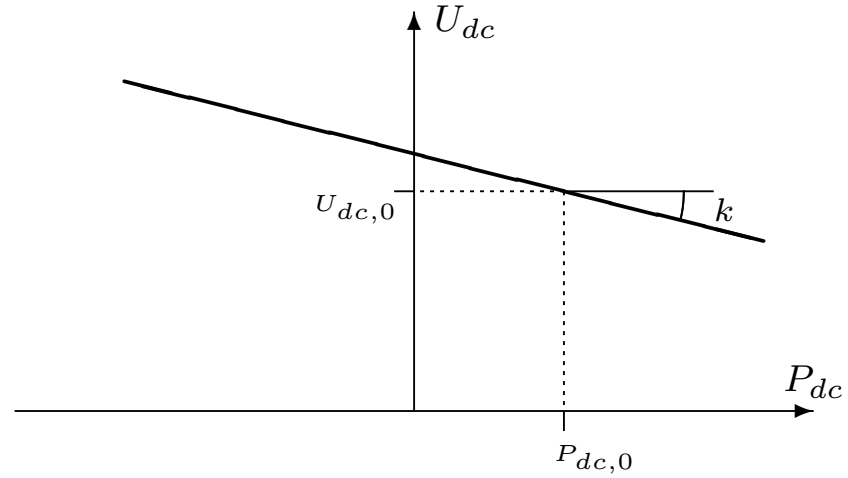

Fig. 2. Standard voltage droop characteristic.

2) $U_{d c}$-control: The converter adapts its active power injection $P_{d c}$ to control its DC bus voltage.

This two-terminal concept can be extended to a VSC HVDC grid by having all-but-one converters fixing their active power injection. The function of the remaining converter is to control the voltage at the DC bus to its reference value, thereby controlling the DC grid voltage profile by clamping the voltage at one bus. From a power flow point of view, there is no objection to assign a voltage control function as described above to more than converter, thereby having multiple slack buses in one DC grid. One of the problems, however, is that this control may give rise to unwanted voltage and power oscillations.

Alternatively, the DC voltage can be controlled by introducing a voltage droop, as shown in Fig. 2. By doing so, multiple converters can assist the voltage control by adapting the power according to their droop characteristic. The lower the value of the voltage droop $k$, with $k$ the opposite of the slope in Fig. 2, the more the converter adapts its output power when the voltage changes. The limit values of the voltage droop $k$ are 0 and $\infty$, at which the converter respectively controls the DC voltage (DC slack) or the DC power ( $P$-control) to their reference values.

The DC voltage droop control shows many similarities to the frequency droop used in $\mathrm{AC}$ systems. However, there are peculiarities which make the DC voltage droop implementation less straight-forward than the frequency droop counterpart. Whereas the frequency remains constant in an AC system, the DC voltage differs from one bus to another in a DC system as a result of the steady-state power flows in the DC grid. From this perspective, voltage deviations at different locations in the DC grid as such do not necessarily reflect transient system conditions. They might as well be the result of the power flows in the DC grid or they might be caused by changing operating conditions. In light of this, [9] suggested to introduce a Load Reference Set Point for each converter, representing the target no-load DC voltage for all converters connected to the grid. Similarly, the implementation put forward in this paper uses a reference voltage $U_{d c, 0}$ and power $P_{d c, 0}$ for the droopcontrolled converters, as shown in Fig. 2. In this paper, these reference values are set to the system's working conditions in steady-state, without a droop control being active. In this way, the power flows are the same as those in a situation with a centralised DC slack bus. After a contingency in the DC 
grid, the system will start operating in a new operation point determined by the voltage droop characteristics. The droop control can be extended to include current and voltage limits. If the dynamic characteristics of the droop control are of interest, the basic droop characteristic from Fig. 2 has to be extended to include a dead band around the set-point voltage value, similar to the frequency droop implementation in governors. Such a detailed representation is out of the scope of this study. Instead, the basic droop characteristics from Fig. 2 are used in the remainder of the paper.

\section{DC GRID POWER FLOW}

This section discusses the DC grid power flow equations and the modifications to the algorithm presented in [8] when a voltage droop is introduced. The power flow equations to solve a DC grid show many similarities to those of a conventional $\mathrm{AC}$ power flow and the methods used to solve the equations are also applied here.

In an AC system, the active power through the lines is mainly linked with the angle difference between different buses, whereas the magnitude of the voltage at the different buses is linked to the flow of reactive power. In DC grids, on the contrary, the power flows are dictated by the differences in voltage magnitude between the different DC buses. The current injected at a DC node $i$ can be written as the current flowing to the other $n-1$ nodes in the network:

$$
I_{d c_{i}}=\sum_{\substack{j=1 \\ j \neq i}}^{n} Y_{d c_{i j}} \cdot\left(U_{d c_{i}}-U_{d c_{j}}\right),
$$

with $Y_{d c_{i j}}$ equal to $1 / R_{d c_{i j}}$.

Combining all currents injected in an $n$ bus DC network results in

$$
\boldsymbol{I}_{\boldsymbol{d c}}=\mathrm{Y}_{\mathrm{dc}} \boldsymbol{U}_{\boldsymbol{d c}}
$$

with the DC current vector $\boldsymbol{I}_{d c}$ given by

$$
\boldsymbol{I}_{d c}=[\underbrace{I_{d c_{1}}, I_{d c_{2}} \ldots I_{d c_{k}}}_{\text {working converters }}, \underbrace{0 \ldots 0}_{\text {outage }}]^{T},
$$

with $n-k$ zero elements due to converter outages and DC buses without a power injection. The DC voltage vector is given by $\boldsymbol{U}_{d c}=\left[U_{d c_{1}}, U_{d c_{2}} \ldots U_{d c_{n}}\right]^{T}$ and $\mathrm{Y}_{\mathrm{dc}}$ is the DC bus matrix. When line outages are taken into account, this involves altering the DC bus matrix accordingly. For a monopolar, symmetrically grounded DC grid, the power injections become

$$
P_{d c_{i}}=2 U_{d c_{i}} I_{d c_{i}}, \quad \forall i \leq k .
$$

A similar expression holds for a bipolar configuration when in steady-state operation.

The current injections $\boldsymbol{I}_{\boldsymbol{d c}}$ are unknown prior to the DC grid power flow. Instead, the active power injections $\boldsymbol{P}_{\boldsymbol{d c}}$ of the converters in $P$-control are calculated using the results of the AC power flow. However, the DC slack bus active power injection $P_{d c}$ is not known prior to the DC power flow. When there are converters with a distributed voltage control, the active power injection $P_{d c}$ at these buses is not known prior to the DC power flow either.

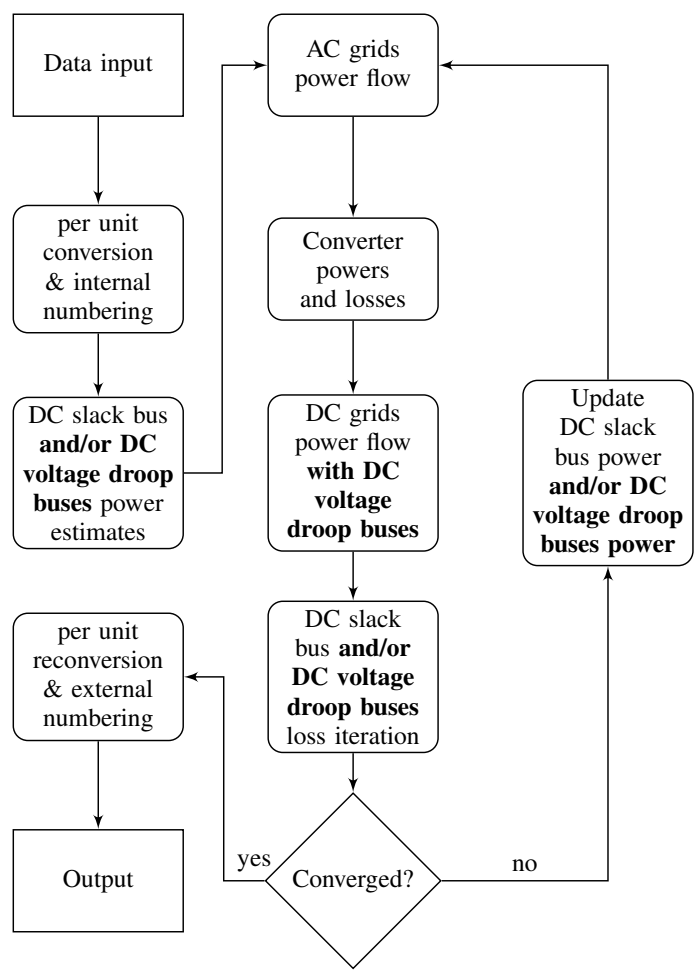

Fig. 3. Flow chart of the sequential VSC AC/DC power flow algorithm with a distributed voltage control.

Combining (2) and (4) and assuming a monopolar, symmetrically grounded DC grid, the known active power injections can be written as

$$
P_{d c_{i}}=2 U_{d c_{i}} \sum_{\substack{j=1 \\ j \neq i}}^{n} Y_{d c_{i j}} \cdot\left(U_{d c_{i}}-U_{d c_{j}}\right) .
$$

The active power injected by the converters in voltage control depends on the deviation of the bus voltage from its reference value and is an unknown prior to the power flow. Using Fig. 2 , the active power injection can be written as

$$
P_{d c_{i}}=P_{d c, 0_{i}}-\frac{1}{k_{i}}\left(U_{d c_{i}}-U_{d c, 0_{i}}\right),
$$

with $k_{i}$ the voltage droop, defined as $\Delta U_{d c} / \Delta P_{d c}$.

This system of non-linear equations can be solved with a Newton-Raphson (NR) method, as shown in the next section.

\section{Sequential power Flow With Distributed DC VOLTAGE CONTROL}

In this section, the DC grid model with a distributed voltage control is introduced in the sequential AC/DC power flow algorithm from [8]. Fig. 3 shows the flow chart of the power flow algorithm when a distributed voltage control scheme is included. The modifications to the sequential power flow algorithm from [8] are printed in bold. In [8], one converter was assigned the task of the DC slack converter, adapting its power to control the DC system voltage. When a DC voltage droop is applied by other converters, it is still possible, but not necessary, to include a slack bus in the DC grid. From a control point of view, this stems with a converter that controls its DC 
bus voltage to the reference value using a PI controller, whilst the converters with a DC voltage droop use a proportional gain. In this section, the equations are written in their most general form, with the first converter being a regular DC slack converter.

The remainder part of the section only briefly discusses the sequential algorithm as such. Instead, emphasis is put on the modifications introduced by to the distributed voltage control.

\section{A. AC grid power flow}

Similarly to the converters under active power control, the DC voltage controlling buses are introduced in the $\mathrm{AC}$ power flow algorithm as either PQ or PV buses. When no generator is present at the $\mathrm{AC}$ bus under consideration, a dummy generator is added to the bus to deliver or absorb the reactive power needed to keep up the AC system voltage. When under constant reactive power control, the reactive part of the load is changed to include the converter's reactive power injection.

All active power injections to the $\mathrm{AC}$ grid are included for the AC power flow calculation by adapting the active part of the load at the selected buses. With the active power injection defined with respect to the AC system, the powers can be modified at once. However, when a converter is under DC voltage control, either a slack bus or droop-based control, the active power injection in the AC grid is not known beforehand, since it depends on the active power needed at the DC side to control the DC voltage. The voltage droop buses can thus be treated in a way similar to the slack converter with regards to the AC system.

As a first estimate to initiate the overall iteration, the $\mathrm{AC}$ active power injections of the droop controlled converters are put equal to the negative of the DC power reference $P_{d c, 0}$, thereby assuming that the DC voltage does not deviate from the reference value $U_{d c, 0}$ and neglecting the converter losses $P_{\text {loss }}$. Without lack of generality, we assume a $n$ bus DC system with the first converter as the DC slack bus and the subsequent $m-1$ converters using a DC voltage droop control. The next $k-m$ buses are under constant active power control. The remaining $n-k$ buses do not have a connection to the AC grid or are facing a converter outage. The active power injection estimate of the $m-1$ converters under voltage control is given by

$$
P_{s_{i}}^{(0)}=-P_{d c, 0_{i}} \quad \forall i: 2 \leq i \leq m .
$$

The active power delivered by the DC slack bus, if present, is initiated as

$$
P_{s_{1}}^{(0)}=-\sum_{i=2}^{m} P_{s_{i}}^{(0)}-\sum_{j=m+1}^{n} P_{s_{j}}
$$

with $P_{s_{i}}^{(0)}$ from (7) and $P_{s_{j}}$ defined prior to the power flow.

After calculating the AC power flow, all converter powers and losses are calculated to obtain the DC grid's injected powers $\boldsymbol{P}_{\boldsymbol{d c}}$ for the $k$ DC buses to which converters are connected, disregarding the ones facing outages.

$$
P_{d c_{i}}=-P_{c_{i}}-P_{l o s s_{i}}, \quad \forall i<k,
$$

with $P_{c}$ the active part of the complex power injected at the converter side, shown in Fig. 1.

During the iteration, this expression does not hold for the DC slack converter and the $m-1$ converters under DC voltage droop for reasons explained above. However, the intermediate AC grid states can be used for estimating the losses of these converters to start the iteration that follows the DC power flow, shown in Fig. 3.

\section{B. DC grid power flow}

With the DC power injections calculated as a result of the AC power flow, a NR iteration, based on (5)-(6) is used to calculate the DC grid's power flow. For the converters under constant power control, $m+1$ to $k$, the DC power injection $P_{d c}$ as defined by (5), is known as a result of the AC power flow. For the converters under distributed voltage control, 2 to $m$, the DC power injection set-points $P_{d c, 0}$ are all known. A modified active power vector $\boldsymbol{P}_{\boldsymbol{d c}}^{\prime}$ is introduced to group these variables, hence

$$
\boldsymbol{P}_{d c}^{\prime}=[\underbrace{P_{d c_{1}}}_{\text {slack }}, \underbrace{P_{d c, 0_{2}} \ldots P_{d c, 0_{m}}}_{\text {voltage droop }}, \underbrace{P_{d c_{m+1} \ldots P_{d c_{k}}}}_{P-\text { control }}, \underbrace{0 \ldots 0}_{\text {outage }}]^{T} .
$$

Using this modified power vector, the DC bus voltages are calculated with a NR method:

$$
\left(\boldsymbol{U}_{\boldsymbol{d} \boldsymbol{c}} \frac{\partial \boldsymbol{P}_{\boldsymbol{d} \boldsymbol{c}}^{\prime}}{\partial \boldsymbol{U}_{\boldsymbol{d c}}}\right)^{(j)} \cdot \frac{\boldsymbol{\Delta} \boldsymbol{U}_{\boldsymbol{d c}}}{\boldsymbol{U}_{\boldsymbol{d c}}}{ }^{(j)}=\boldsymbol{\Delta} \boldsymbol{P}_{\boldsymbol{d c}}^{\prime(j)}
$$

The equations and terms corresponding to the slack bus are removed since its voltage is known prior to the DC network power flow.

In this system of equations, the modified power mismatch vector $\Delta \boldsymbol{P}_{\boldsymbol{d c}}^{\prime(j)}$ is given by

$$
\Delta P_{d c_{i}}^{(j)}=\left\{\begin{array}{cl}
P_{d c, 0_{i}}-P_{d c, 0_{i}}\left(\boldsymbol{U}_{\boldsymbol{d c}}{ }^{(j)}\right) & \forall i: 2 \leq i \leq m \\
P_{d c_{i}}^{(k)}-P_{d c_{i}}\left(\boldsymbol{U}_{\boldsymbol{d c}}{ }^{(j)}\right) & \forall i: m<i \leq k \\
-P_{d c_{i}}\left(\boldsymbol{U}_{\boldsymbol{d c}}{ }^{(j)}\right) & \forall i: k<i \leq n
\end{array}\right.
$$

with $P_{d c, 0_{i}}\left(\boldsymbol{U}_{\boldsymbol{d c}}{ }^{(j)}\right)$ given by

$$
P_{d c, 0_{i}}^{(j)}=P_{d c_{i}}\left(\boldsymbol{U}_{\boldsymbol{d c}}{ }^{(j)}\right)+\frac{1}{k_{i}}\left(U_{d c_{i}}^{(j)}-U_{d c, 0_{i}}\right),
$$

and superscripts $(j)$ and $(k)$ respectively referring to the inner NR iteration and the outer AC/DC power flow iteration. The terms of the Jacobian are given by

$$
\begin{aligned}
& \left(U_{d c_{j}} \frac{\partial P_{d c_{i}}}{\partial U_{d c_{j}}}\right)^{(j)}=-2 U_{d c_{i}}^{(j)} Y_{d c_{i j}} U_{d c_{j}}^{(j)} \\
& \left(U_{d c_{i}} \frac{\partial P_{d c_{i}}}{\partial U_{d c_{i}}}\right)^{(j)}=P_{d c_{i}}^{(j)}+2 U_{d c_{i}}^{(j)^{2}} \sum_{\substack{j=1 \\
j \neq i}}^{n} Y_{d c_{i j}}, \\
& \left(U_{d c_{j}} \frac{\partial P_{d c, 0_{i}}}{\partial U_{d c_{j}}}\right)^{(j)}=\left(U_{d c_{j}} \frac{\partial P_{d c_{i}}}{\partial U_{d c_{j}}}\right)^{(j)}, \\
& \left(U_{d c_{i}} \frac{\partial P_{d c, 0_{i}}}{\partial U_{d c_{i}}}\right)^{(j)}=\left(U_{d c_{i}} \frac{\partial P_{d c_{i}}}{\partial U_{d c_{i}}}\right)^{(j)}+\frac{1}{k_{i}} U_{d c, i}^{(j)}
\end{aligned}
$$




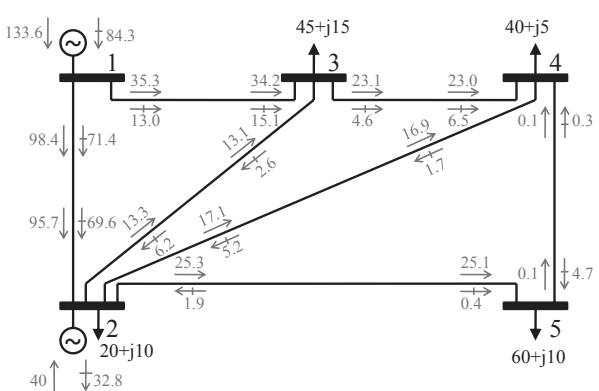

(a) Normal operation

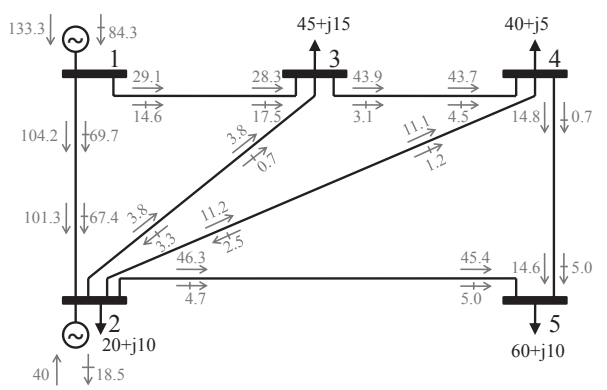

(b) Outage: $P$-control

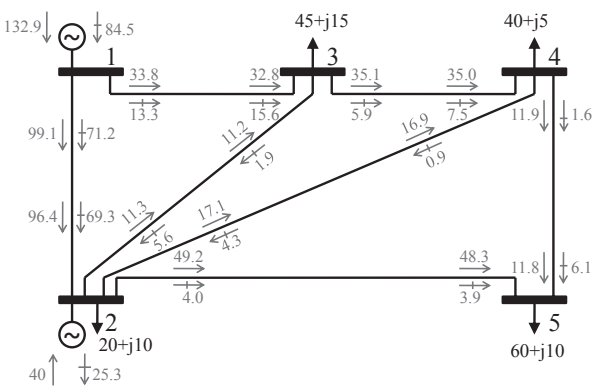

(c) Outage: DC voltage droop

Fig. 4. AC network power flow solution with a VSC MTDC system between buses 2, 3 and 5: (a) Normal operation, (b) Converter outage on bus 5 \& $P$-control, (c) Converter outage on bus $5 \&$ DC voltage droop on converters 2 and 3. Legend: $\rightarrow$ Active power $(M W)$ and $\nrightarrow$ Reactive power $(M V A r)$.

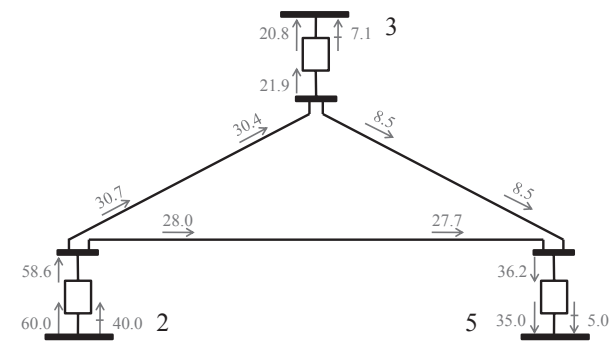

(a) Normal operation

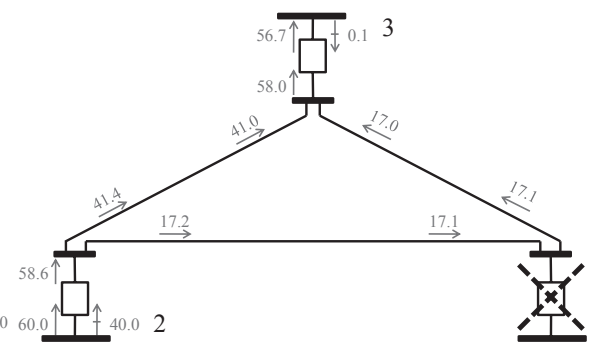

(b) Outage: $P$-control

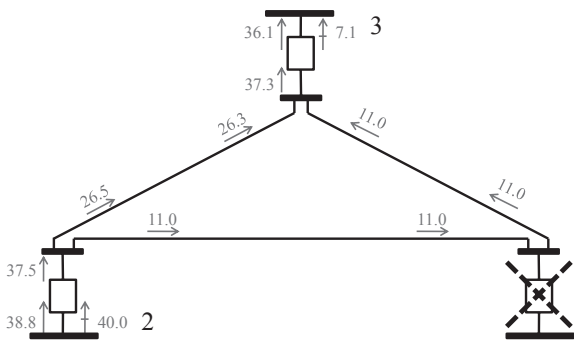

(c) Outage: DC voltage droop

Fig. 5. DC network power flow solution with a VSC MTDC system between buses 2, 3 and 5: (a) Normal operation, (b) Converter outage on bus 5 \& $P$-control, (c) Converter outage on bus $5 \&$ DC voltage droop on converters 2 and 3. Legend: $\rightarrow$ Active power $(M W)$

After convergence, the voltages on all DC buses are known. Thereafter, the power injections of the slack bus and the DC voltage droop buses are calculated using (5).

\section{Additional converter loss iteration}

After having calculated all unknown DC grid voltages and powers, an additional iteration is needed to calculate the $\mathrm{AC}$ grid power injection of the DC slack converter and the voltage droop converters since the AC powers injected by these converters depend on the converter losses, which are not known beforehand.

Due to the extension of the algorithm, the overall convergence criterium has to be based on the slack converter power, if present, as well as on the voltage droop buses' power injections in the AC grid, as shown in Fig. 3.

\section{Simulation Results}

This section discusses the effect of a converter outage on the power flows in the AC and DC grid. The extension of the sequential power flow algorithm allows to study the effect of the droop characteristics on the overall power flows after a disturbance.

The DC voltage droop control has been included in a sequential AC/DC power flow algorithm, using MATPOWER [10], an open-source power flow toolbox for Matlab. The simulation results, shown in Figs. $4-5$, are based on a 5-bus test network from [11] with a 3-bus VSC MTDC network. Figs. 4a \& 5a show the results of the AC and DC test system under normal operation conditions. Converters on buses 2 and
5 are under constant $P$ and $Q$-control. The converter at bus 3 is under constant $U$-control and is also designated the role of the DC slack converter. The DC voltages and DC power injections from this power flow are used as the reference values for the DC voltage droop control later on. Figs. $4 \mathrm{~b} \& 5 \mathrm{~b}$ show the results of the AC and DC test system with a converter outage on bus 5. As expected, the power of the slack converter at bus 3 adapts its power, whereas the power injected by the converter at bus 2 remains unaltered. In Figs. $4 \mathrm{c} \& 5 \mathrm{c}$, a voltage droop control has been implemented on all converters. The converters remaining in operation, namely those on buses 2 and 3 have a voltage droop that has been respectively set to 5e-3 and 7e-3 p.u./MW. As shown by the results, the remaining converter powers are both lowered, dictated by their droop characteristics. As expected, the droop control also alters the power flows in the AC grid, shown in Fig. 4.

When the droop control is implemented in a larger DC network, the contribution of each converter to the DC voltage control can be adapted by altering its droop characteristic.

\section{CONCLUSION}

In this paper, a model has been presented to include voltage droop characteristics in power flow calculations. The model has been implemented and tested in a sequential AC/DC power flow algorithm. The implementation of the droop characteristics allows to extend contingency analyses to DC grids and makes it possible to study the effects of the distributed voltage control and the individual droop values of each converter on the post-disturbance power flows in both the AC and DC system. The droop characteristics have been included in a 
MATPOWER based AC/DC power flow program and properly tested. Numerical simulations show the effect of the distributed voltage control on the power flows in the system.

\section{REFERENCES}

[1] D. Van Hertem and M. Ghandhari, "Multi-terminal VSC HVDC for the European supergrid: Obstacles," Renewable and Sustainable Energy Reviews, vol. 14, no. 9, pp. 3156-3163, Dec. 2010.

[2] C. Dierckxsens, "VSC MTDC systems with distributed slack bus," Master's thesis, K.U.Leuven, Jun. 2010.

[3] R. Hendriks, G. Paap, and W. Kling, "Control of a multi-terminal VSC transmission scheme for connecting offshore wind farms," in Proc. European Wind Energy Conference \& Exhibition, Milan, Italy, May, 7 - 102007.

[4] T. Haileselassie and K. Uhlen, "Primary frequency control of remote grids connected by multi-terminal hvdc," in Proc. IEEE PES GM '10, Minneapolis, USA, Jul. 25-29, 2010.

[5] A. van der Meer, R. Hendriks, and W. Kling, "Combined stability and electro-magnetic transients simulation of offshore wind power connected through multi-terminal VSC-HVDC," in Proc. IEEE PES GM '10, Minneapolis, USA, Jul. 25-29, 2010, pp. $1-7$.

[6] T. Haileselassie, K. Uhlen, and T. Undeland, "Control of multiterminal HVDC transmission for offshore wind energy," in Proc. Nordic Wind Power Conference Presentation, Rønne, Denmark, Sep. 10-11, 2009.

[7] G. Asplund, B. Jacobson, B. Berggren, and K. Lindén, "Continental overlay HVDC-grid," in CIGRE 2010 Session Proceedings, Paris, France, 2010.

[8] J. Beerten, S. Cole, and R. Belmans, "Generalized steady-state VSC MTDC model for sequential AC/DC power flow algorithms," IEEE Trans. Power Syst., 2011, submitted for publication.

[9] C. Barker and R. Whitehouse, "Autonomous converter control in a multiterminal HVDC system," in Proc. IET ACDC '10, London, UK, Oct. 20-21, 2010.

[10] R. D. Zimmerman, C. E. Murillo-Sanchez, and R. J. Thomas, "MATPOWER: Steady-state operations, planning, and analysis tools for power systems research and education," IEEE Trans. Power Syst., vol. 26, no. 1 , pp. 12-19, Feb. 2011

[11] G. W. Stagg and A. H. El-Abaid, Computer Methods in Power System Analysis. Kogakusha, Japan: McGraw-Hill, 1968.

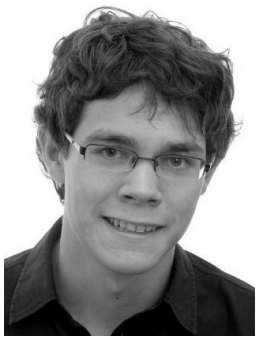

Jef Beerten (S'07) was born in Belgium in 1985. $\mathrm{He}$ received the M.Sc. degree in electrical engineering from the Katholieke Universiteit Leuven (K.U.Leuven), Leuven, Belgium, in 2008, where he is currently working towards the Ph.D. degree. He is a Research Assistant with the division ESATELECTA division of K.U.Leuven. His research interests include power system control, integration of wind energy, DC grids and multi-terminal VSC HVDC in particular. Mr. Beerten is an active member of both the IEEE and Cigré

Mr. Beerten holds a Ph.D. fellowship from the Research Foundation Flanders (FWO).

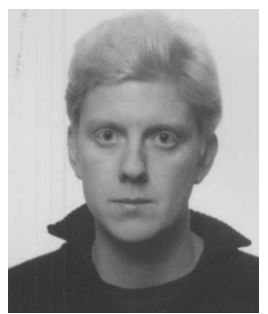

Dirk Van Hertem (S'02, SM'09) graduated as a M.Eng. (industrieel ingenieur) in Electro-mechanical engineering in 2001 from the KHK, Geel, Belgium and as a M.Sc.(burgerlijk ingenieur) in Electrical Engineering from the K.U.Leuven, Belgium in 2003. In January 2009, he obtained his $\mathrm{PhD}$, also from the K.U.Leuven. From 2010 to spring 2011, Dirk Van Hertem was a member of the Electric Power Systems division at the Royal Institute of Technology, Stockholm. Currently, he is working again as a postdoctoral researcher in the ELECTA research group at the K.U.Leuven, Belgium.

His special fields of interest are power system operation and control in systems with FACTS and HVDC and building the (super-)grid of the future with large amounts of renewable energy sources. Dr. Van Hertem is an active member of both IEEE and Cigreé.

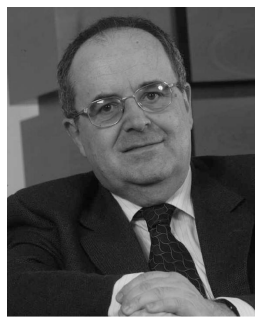

Ronnie Belmans (S'77-M'84-SM'89-F'05) received the M.Sc. degree in electrical engineering in 1979 and the Ph.D. degree in 1984, both from the K.U.Leuven, Belgium, the Special Doctorate in 1989 and the Habilitierung in 1993, both from the RWTH, Aachen, Germany. Currently, he is a full professor with the K.U.Leuven, teaching electric power and energy systems. His research interests include techno-economic aspects of power systems, power quality and distributed generation. He is also guest professor at Imperial College of Science, Medicine and Technology, London-UK. 